\title{
Dietary Patterns and Cognitive Health in Older Adults: A Systematic Review
}

Xia Chen, Brook Maguire, Henry Brodaty, Fiona O'Leary

[Journal of Alzheimer's Disease, 67(2) 2018, 583619, DOI 10.3233/JAD-180468]

https://content.iospress.com/articles/journal-ofalzheimers-disease/jad180468

On page 606, the last two sentences in second paragraph under "Mediterranean diet", starting from "After a median follow up of 4.1 years" should be:

"Updated results published in 2018 for the Barcelona study, confirmed that after a median follow up of 4.1 years, the Mediterranean diet plus nuts group had significantly improved memory composite, while Mediterranean diet plus EVOO group had significantly better performance on the frontal and global cognition composites compared to the control group. However, after reanalysis [66], no between-group differences were observed for individual cognitive tests after adjustment for confounders in multivariate analysis."

[66] Agarwal A, Ioannidis JPA (2019) PREDIMED trial of Mediterranean diet: retracted, republished, still trusted? BMJ 364, 1341.

On Page 606 under "Mediterranean diet", the last sentence in first paragraph "Both studies had high risk of bias due to the difficulty of blinding", should instead be:

"Both studies had high risk of bias due to the difficulty of blinding $[66,67]$ as well as randomization issues in one trial [65]"
On page 585 Table 1, in the row of "Valls-Pedret et al [65]", Quality assessment should be:

\footnotetext{
"Random Sequence Generation"- high

"Allocation concealment"-high

"Selective reporting"- low

"Other source of bias"- yes

"Blinding- participants and personnel"- high

"Blinding-outcome assessment"- low

"Incomplete outcome data"- high

"Overall bias assessment"- high
}

On page 587 Table 2, in the row of "Valls-Pedret et al 2015 [65]", Key findings should be:

"No between-group differences were observed for cognitive tests after adjusted for confounders in multivariate analysis. Memory composite score $(n=334)$ was higher in the Mediterranean diet plus nuts 0.10 (95\%CI: -0.04 to 0.24, $P=0.04$ ); frontal cognition composite score $(n=96)$ was higher in the Med Diet plus EVOO 0.23 (95\% CI: $0.02,0.43, P=0.004)$; global cognition composite $(n=96)$ was higher in the Med Diet plus EVOO 0.04 (95\%CI: $-0.12,0.20, P=0.008$ ) compared to controls. All cognitive composite scores significantly $(P<0.05)$ decreased from baseline in controls."

On page 617 Reference [65] should be:

[65] Valls-Pedret C, Sala-Vila A, Serra-Mir M, Corella D, de la Torre R, Martinez-Gonzalez MA, Martinez-Lapiscina EH, Fito M, PerezHeras A, Salas-Salvado J, Estruch R, Ros E (2015) Mediterranean diet and age-related cognitive decline: A randomized clinical trial. JAMA Intern JAMA Intern Med. 2015;175(7):10941103. doi:10.1001/jamainternmed.2015.1668 
Published online May 11, 2015. Corrected on November 5, 2018.

On page 606 and references page 617, new references added are:

[66] Agarwal A, Ioannidis JPA (2019) PREDIMED trial of Mediterranean diet: retracted, republished, still trusted? BMJ 364, 1341.
[67] Martínez-González MA (2018) Protocol Deviations, Reanalyses, and Corrections to Derivative Studies of the PREDIMED Trial. JAMA Intern Med 178, 1730-1731. 